Génétique des textes littéraires - Afrique, Caraïbe, diaspora

\title{
Retouches de la représentation de la femme dans trois éditions de la nouvelle de Joseph Zobel «Pionnier d'une nuit » (ou « Le premier convoi »)
}

Simples lissages stylistiques, expressions d'une maturation psychologique de l'écrivain, ou révisions socio-culturelles?

Charles W. Scheel

\section{(2) OpenEdition}

Édition électronique

URL : https://journals.openedition.org/coma/5012

DOI : 10.4000/coma.5012

ISSN : 2275-1742

Éditeur

Institut des textes \& manuscrits modernes (ITEM)

Référence électronique

Charles W. Scheel, « Retouches de la représentation de la femme dans trois éditions de la nouvelle de Joseph Zobel «Pionnier d'une nuit » (ou « Le premier convoi ») », Continents manuscrits [En ligne], 13। 2019, mis en ligne le 22 novembre 2019, consulté le 14 janvier 2023. URL : http:// journals.openedition.org/coma/5012 ; DOI : https://doi.org/10.4000/coma.5012

Ce document a été généré automatiquement le 14 janvier 2023.

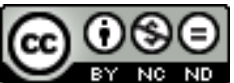

Creative Commons - Attribution - Pas d'Utilisation Commerciale - Pas de Modification 4.0 International - CC BY-NC-ND 4.0

https://creativecommons.org/licenses/by-nc-nd/4.0/ 


\section{Retouches de la représentation de la femme dans trois éditions de la nouvelle de Joseph Zobel « Pionnier d'une nuit » (ou « Le premier convoi »)}

Simples lissages stylistiques, expressions d'une maturation psychologique de l'écrivain, ou révisions socio-culturelles?

\section{Charles W. Scheel}

L'histoire assez complexe de l'édition des œuvres littéraires de Zobel a été évoquée dans deux ouvrages récents. L'un porte sur le rapport de Zobel avec la négritude dans l'ensemble des six romans publiés ${ }^{1}$, et l'autre sur les premières publications de contes et de reportages de Zobel dans l'hebdomadaire Le Sportif de Fort-de-France entre 1938 et $1959^{2}$. Si les textes des romans les plus connus de Zobel, Diab'-là ${ }^{3}$ et La Rue CasesNègres ${ }^{4}$, ont très peu varié au gré de leurs éditions, on sait dorénavant bien mieux, grâce à l'ouvrage de Louise Hardwick, que des modifications plus substantielles sont à la base de la réédition des Jours immobiles (Fort-de-France, 1946) sous le titre Les Mains pleines d'oiseaux (Paris, Nouvelles Éditions Latines, 1978) et celle de La Fête à Paris (Paris, La Table Ronde, 1953) sous le titre nouveau Quand la neige aura fondu (Paris, Éditions Caribéennes, 1979).

2 Or c'est dans cette même période de la fin des années 1970 que le jeune retraité de Radio Sénégal, Joseph Zobel, désormais revenu en France et installé à demeure dans sa maison des Cévennes, a également révisé son premier recueil de nouvelles : Laghia de la mort qui était paru initialement à compte d'auteur à Fort-de-France en 1946, reparaît chez Présence Africaine en 1978, après trente deux ans de sommeil. La nouvelle édition conserve sept des huit récits d'origine ${ }^{5}$ mais avec d'importantes modifications dans les textes : «Laghia de la mort » (drame troublant de la filiation, exprimé dans un combat de damier/laghia opposant un père et son fils - ce dernier venant seulement de 
découvrir l'identité du premier qui ne l'avait jamais reconnu ni légalement ni socialement ${ }^{6}$ ); "Défense de danser " (à propos d'une fête de Noël dans la tradition antillaise, au cours de laquelle Borès, l'ancien amant de Denise, un mulâtre, ne veut pas lui permettre de danser avec Rigobert, un "nègre étranger ", venu d'un village voisin pour la coupe de la canne) ; « Le premier convoi » (intitulé précédemment « Le pionnier d'une nuit ", sur une nuit d'amour à Fort-de-France entre Ferdinise et Isidore, un soldat mobilisé pour la guerre) ; «Coup de nuit » (l'aventure du nègre Léonal qui rentre chez lui de nuit, dans la campagne, après avoir gagné une grosse somme aux cartes contre des inconnus dont il craint qu'ils ne le suivent pour le dévaliser); "Le syllabaire » (l'histoire de l'humiliation vécue par le petit Aristide et sa mère Théodamise, sarcleuse de cannes à Petit Bourg, qui ont fait un long chemin un dimanche matin pour acheter un manuel d'école que la vendeuse n'a pas voulu céder car il leur manquait quinze centimes) ; «Il était un petit navire » (le souvenir d'une rencontre galante avec le beau marin Jimmy de Trinidad, qui revient en mémoire à Émilienne, lors d'une promenade dans le port de Fort-de-France en période de guerre) ; et « Mapiam » (l'histoire du petit Casimir Mbafo qui essaie d'éviter une humiliation devant sa classe alors que sa mère ne peut plus lui payer de souliers).

3 Trois des protagonistes principaux de ces contes sont des femmes, mais elles sont quasiment inconnues du public de lecteurs de Zobel, en raison de la popularité extraordinaire des héros du roman La Rue Cases-Nègres : le jeune José Hassam et surtout sa vaillante grand-mère, M'man Tine. Or un autre personnage féminin, annonciateur de certaines qualités de M'man Tine, dominait déjà le second roman de Zobel, Les Jours immobiles, qui met en scène le pêcheur Géo et sa grand-mère Cocotte dans la commune des Anses d'Arlet - non loin de celle du Diamant, cadre du roman précédent, Diab'-là ${ }^{7}$.

4 Tout aussi négligées par la critique que Cocotte, sont donc les nombreuses figures féminines dans les contes ou nouvelles ${ }^{8}$ de Zobel, dont plusieurs, dès le premier recueil, Laghia de la mort, sont représentées sous un angle étonnamment moderne pour l'époque et le contexte antillais. C'est le cas de Ferdinise dans le "Pionnier d'une nuit", d'Émilienne dans "Il était un petit navire", ainsi que de Denise dans "Défense de danser $"$. Chacune de ces protagonistes serait - aux yeux de lecteurs bien-pensants une variante du personnage de "la femme tombée », mais le narrateur omniscient des textes les représente - souvent en focalisation interne, d'ailleurs - sans l'ombre d'une jugement moral. Une étude des retouches apportées à la représentation de ces femmes dans les trois récits, en tenant compte du contexte biographique et socio-culturel de leur rédaction et/ou parution, serait intéressante. Mais on se limitera ici au premier que Zobel a retenu pour parution dans son recueil Laghia de la mort ${ }^{10}$.

5 Avec des protagonistes comme Ferdinise et Émilienne, Zobel intègre dans sa représentation de la Martinique contemporaine, les bouleversements que la guerre venait susciter dans les rapports hommes-femmes sur l'île. Tout cela était encore d'une grande actualité lorsque le jeune auteur a choisi de réunir certains de ces textes dans le recueil Laghia avant de quitter la Martinique pour la métropole en novembre 1946. Cela ne l'était évidemment plus du tout lors de la révision entreprise trente ans plus tard. Et l'on peut se demander qui - des personnages ou de l'auteur - a vieilli davantage dans cette réécriture.

6 Pour répondre à cette question pour le seul récit du "Pionnier d'une nuit » (transformé en «Le premier convoi » dans l'édition de 1978), les trois versions publiées (étiquetées chronologiquement $\mathrm{A}, \mathrm{B}$ et $\mathrm{C}$ ) ont été juxtaposées page par page dans 
l'annexe 1. Toutes les modifications n'ont pas été répertoriées, mais des mots et des expressions-clés ont été mis en gras pour faciliter le repérage de jalons dans le récit, et les variations de leur distribution dans les différentes versions. D'autre part, ont été surlignés en rose les mots ou groupes de mots qui ont été supprimés dans la version suivante, et en bleu ceux qui ont été rajoutés - soit pour remplacer les excisions soit pour étoffer le récit. On peut ainsi constater d'emblée que l'œuvre n'évolue presque pas entre les versions $A$ et $B$, mais bien davantage entre $B$ et $C$.

\section{Lissages stylistiques}

$7 \quad$ La part des modifications purement stylistiques d'une version à l'autre est assez limitée et relève souvent de la simple préférence lexicale pour un autre verbe, nom, adjectif, préposition ou syntagme synonyme, qui n'affecte guère le sens des phrases ou l'interprétation de l'histoire. Ainsi, alors qu'au début des versions A et B, la jeune femme est « réjouie » de la question du soldat, elle est « amusée » dans la version C (1). De façon similaire les " godasses » du soldat de la version A deviennent des « godillots » (terme moins argotique et plus technique, en contexte militaire) dans les versions B et C (3), et les « images saintes » autour de la lampe de la chambre, dans les versions A et B (2), deviennent des «images de piété » dans la version C (3). Plus loin dans le texte, Ferdinise «se mit à sangloter» dans la version $\mathrm{A}$, mais «fondit en larmes » dans la version $B$ (A et $B, 7$ ), deux formulations différentes, certes, mais pratiquement équivalentes sur le plan sémantique.

Des modifications d'un seul mot peuvent pourtant modifier un portrait en introduisant une autre connotation. Ainsi, le pionnier, décrit comme " grand, large et fort » au début des deux premières versions, devient-il « grand, alerte et fort » dans la troisième (1). La silhouette d'Isidore s'en trouve affinée et sa personnalité avivée. Un effet similaire est produit dans la même version C qui change le surnom d'Isidore de "Dodore » en « Zido » (3) ; vers la fin de la version B, il est toujours « grand, large et fort » mais aussi «beau!» aux yeux de Ferdinise, alors que dans la version C, elle se souvient de lui simplement comme « grand » et « costaud » (7).

On peut aussi parler d'effet stylistique dans les nombreux passages qui étoffent l'histoire, surtout entre les versions B et C, distantes de trente ans. Ainsi, pour en rester à la page 1 , la vision initiale très synthétique qu'a Isidore d'une Ferdinise à «la silhouette belle et bien pleine » est-elle développée en une longue phrase qui introduit une image sensuelle et "filée » du corps de la jeune femme, comme un "pain doré » qu'Isidore a envie de « presser avec les deux mains pour le plaisir de voir et d'entendre craquer la croûte » $(C, 1)$.

Un travail esthétisant similaire est fait dans plusieurs passages descriptifs des lieux, qui tendent à être précisés ou décrits de manière plus imagée. Ainsi la sortie de Ferdinise vers le marché le lendemain de sa nuit avec Isidore est-elle augmentée de toute une phrase sur "la rue Brithmer", " avec ses véhicules titubant sur les fondrières, ses marchands ambulants, ses gosses dépenaillés, et toutes les scènes dont on eût dit les rites du quartier pour le commencement du jour » $(C, 5)$ et de plusieurs autres sur « la Place de la Mission ", vers laquelle "toute la ville accourait au lieu de fuir [et où] la foule était déjà mêlée comme les eaux de plusieurs affluents d'un fleuve en crue » $(C, 7)$, alors que ces lieux n'avaient pas été nommés dans les versions précédentes. 
11 Mais de tels effets d'embellissement ou d'augmentation se combinent souvent avec des réflexions nouvelles sur les personnages et leurs agissements, qui indiquent une maturation psychologique de l'écrivain et/ou une révision de la représentation des personnages dans le cadre socio-culturel (de l'action et/ou de la rédaction par l'écrivain).

\section{Une maturation psychologique entre les versions?}

12 Le Zobel qui publie « Pionnier d'une nuit » dans Le Sportif en janvier 1940 a vingt-quatre ans. Il est célibataire et enseigne dans un lycée de garçons au centre de Fort-de-France. Il est intéressant de noter que ce "conte » comporte deux mouvements qui sont très évidents dans la première version, celle du journal, car ils se trouvent répartis sur deux pages non consécutives (4 et 6 ), et se terminent chacun sur quasiment la même image. Le premier mouvement (page 4) s'arrête sur l'image de Ferdinise "blottie contre le corps athlétique du soldat » qu'elle vient d'emmener passer la nuit chez elle ${ }^{11}$. Pour le lecteur de cette version $\mathrm{A}$, le début du récit (qui s'arrête avec ":" suivis de la mention «suite p. 6 ») relève de la simple anecdote d'une rencontre érotique entre jeunes adultes consentants, dans le contexte particulier du Fort-de-France contemporain ${ }^{12}$. Or dans le second mouvement du récit (p. 6 dans le jounal), le texte reprend exactement après les deux points, avec une longue tirade de Ferdinise à Isidore, qui commence avec une déclaration d'une solennité assez surprenante, vu la situation d'énonciation :

Blottie contre le corps athlétique du soldat, Ferdinise continua d'une voix faible et câline, avec un accent plein de tressaillements, qui disait plus que ses paroles :

- Si tu veux, chaque soir tu viendras me voir, je t'aimerai comme je t'aime ce soir,

de toute mon âme... (A et B, 3 et 4 )

Cette deuxième partie du récit est développée par le narrateur des versions $\mathrm{A}$ et $\mathrm{B}$ en trois temps: le rêve sentimental de Ferdinise au cours de la nuit et l'au-revoir des amants à l'aube (une page); la recherche désespérée du soldat au matin par Ferdinise, pour lui remettre un cadeau d'adieu - car elle découvre que les soldats mobilisés partent justement ce jour-là, dans une ville chamboulée par l'événement (deux pages et demi) ; puis une espèce d'épilogue avec la recherche subséquente, "naïve et ardente " (A et B , 7) par Ferdinise, de cet amant idéal qu'a été Isidore pour elle, dans les bras d'autres soldats, attendus à la sortie de la caserne (une page). De la simple rencontre initiale d'une nuit entre deux personnes dans une chambre obscure, le récit passe à l'évocation d'une journée fébrile vécue par Ferdinise dans un Fort-de-France innondé de soleil et affolé par le départ des soldats, avant de revenir à la chambre obscure dans un discours itératif sur le désir de Ferdinise de retrouver Isidore dans d'autres bras, et de proposer en clôture une variation de l'image offerte en fin de premier mouvement :

Alors un tressaillement la raidissait dans toute sa chair, et elle plaquait son corps

souple et nerveux contre la poitrine chaude du soldat. (A, 8)

La sentimentalité de la protagoniste est exprimée au style direct dans le long passage qui introduit la partie rajoutée : ce qui séduit Ferdinise, c'est non seulement le physique d'Isidore, mais qu'il soit soldat (ce qui le dispense de lui « donner » quelque chose); de plus, comme il se dit être pêcheur et provenir d'un village, elle le considère comme plus fiable que « les hommes de la ville " qu'elle dit détester; avant de s'endormir dans ses bras, elle lui propose donc de se considérer chez lui, dans cette chambre, quand il reviendra, après la guerre, et lui apportera du poisson. Mais le narrateur omniscient 
relativise immédiatement ce rêve, en distinguant entre "la nuit d'amour et d'ivresse pour Ferdinise $\mathrm{e}^{13}$ » et la "simple nuit de plaisir pour Isidore» (A et B, 4). Cette distinction des perceptions est renforcée par la promesse laconique du soldat de revenir le soir, manifestement pour faire plaisir à Ferdinise qui le supplie « d'avoir pitié de son idéal »: avoir un homme à demeure, puisque le père de son enfant ne s'en occupe pas. Et le lecteur peut imaginer un narrateur peut-être un brin ironique, quand il fait s'exclamer Ferdinise : «Le bon soldat!» après sa découverte sur la table d'un «billet de cent sous".

Avec ce détail, Zobel dévoile l'aspect vénal de la nuit d'amour - aspect que Ferdinise se cache, préférant voir dans ce billet un cadeau (généreux). Mais cette illusion est détruite plus tard, en ville, par un soldat à qui elle demande s'il connaitt Isidore, et qui lui répond : "Eh bien, ma doudou, dans les conditions qu'on part, s'il ne t'a pas payée hier soir, ton Zidore !...» (A et $\mathrm{B}, 6)$. Cette remarque, d'un réalisme cynique, intime donc que ces nuits « d'amour » entre soldats et jeunes femmes relève d'une pratique au moins tacitement - tarifée, au grand choc de Ferdinise, qui, «brutalement atteinte dans son espérance », «fondit en larmes » $(\mathrm{B}, 6)$.

Ferdinise conserve sa sentimentalité naïve puisqu'elle est décrite, en fin de récit, comme pleurant pour que le soldat «que chaque soir mettait près d'elle [...] l'aimât ${ }^{14}$ au-delà de la guerre", alors qu'elle savait "qu'elle ne pouvait jamais [le] voir plus d'une nuit ». Notons l'ultime petite modification apportée par Zobel en fin de version $\mathrm{B}$ : le narrateur ajoute qu'en plaquant « son corps souple et nerveux contre la poitrine chaude du soldat ", Ferdinise ne faisait qu'essayer de "rattraper un songe fugitif » (B, 7). Quoique très empathique pour sa protagoniste, le narrateur zobélien du « Pionnier d'une nuit » montre donc que Ferdinise demeure très immature dans sa relation avec les hommes ${ }^{15}$.

Quant à Zobel lui-même, lorsqu'il insère la version B de la nouvelle dans Laghia de la mort en 1946, il est marié depuis trois ans et déjà père de deux petits garçons. Il a connu aussi un développement spectaculaire de sa carrière, puisqu'il avait été attaché de presse du Gouverneur Ponton entre septembre 1943 et juillet 1944, et que son premier roman, Diab'-là, avait connu un grand succès dès sa parution à Fort-de-France en 1945. Les petites modifications du texte entre les versions $A$ et $B$ n'indiquent guère de changement dans la maturité de l'écrivain Zobel, sinon dans le commentaire déjà noté plus haut exposant la naïveté du «songe fugitif » que Ferdinise essaie de rattaper chaque soir.

18 L'intérêt de la nouvelle réside dans la représentation empathique des relations entre deux protagonistes à la fois singuliers et représentatifs de types dans un Fort-de-France tourneboulé par l'irruption de la Seconde Guerre mondiale, alors qu'elle se trouve si loin de l'Europe. Intéressant aussi de constater comment ce bouleversement affecte l'écriture de Zobel, puisque l'apprenti conteur qui venait seulement de publier quelques récits dans Le Sportif de Fort-de-France se retrouve apprenti reporter. On vient d'ailleurs de découvrir un bref reportage par Kay-Mac-Zo du départ de ce premier convoi historique des mobilisés de Martinique en novembre 1939 (voir annexe 2) : c'est un poète patriote qui s'y exprime, plutôt qu'un journaliste ${ }^{16}$. En tout cas, les lecteurs de Martinique se souvenaient certainement encore très bien de cet événement lors de la sortie du recueil Laghia de la mort en $1946^{17}$. 


\section{Des révisions socio-culturelles dans la version C?} Africaine en 1978 suscite plusieurs questions ${ }^{18}$. La page de copyright du nouveau recueil ne mentionne pas l'édition de 1946 : il ne s'agit donc pas d'une simple réédition de l'oeuvre imprimée par Bezaudin à Fort-de-France ${ }^{19}$. Comme déjà signalé, la nouvelle édition se distingue surtout par trois aspects : l'absence de la nouvelle «Bo-bo-bo-o »; l'effacement des dédicaces individuelles des huit nouvelles de 1946; et le changement du titre de la nouvelle "Pionnier d'une nuit» en "Le premier convoi». Si ces différences sont facilement apparentes pour qui a en main les deux éditions, les modifications au sein des textes-mêmes le sont beaucoup moins et exigent une lecture attentive. Et si l'on peut estimer que le contenu de la nouvelle édition reprend entre 80 et 90 pour cent de celui de l'ancienne, les changements peuvent être notables. C'est le cas de la nouvelle au centre de la présente étude.

ques variantes d'ordre stylistique ont déjà été mentionnées entre les versions B et $C$, mais elles n'expliquent pas le changement frappant du titre de la nouvelle de «Pionnier d'une nuit » en " Le premier convoi ». Alors que le mot " pionnier » apparaît sous forme d'exclamation dès l'incipit des trois versions, « convoi » n'apparaît que dans la version $\mathrm{C}$, où le mot complète une phrase déjà présente dans les versions $\mathrm{A}$ et $\mathrm{B}$ : «Oh! Elle avait bien entendu parler de ces uniformes qu'on préparait pour ceux qui devaient partir, ceux du premier convoi » (B et C, 5). C'est d'ailleurs le simple mot " partir » que Zobel avait retenu comme titre laconique de son reportage sur l'événement, deux mois plus tôt (voir Annexe 2). Faut-il voir dans cette précision un nouvel effet de « la maturation psychologique» de l'écrivain? Sans doute pas, même si Zobel a évidemment énormément vécu et appris entre 1946 et 1978, période qui inclut dix années de carrière en France puis une vingtaine au Sénégal. Car cette réécriture des nouvelles de jeunesse se fait parallèlement à celle de deux romans : Les Jours immobiles et La Fête à Paris. Or c'est dans ce dernier roman de Zobel, suite des aventures du José Hassam de La Rue Cases-Nègres (mais rédigées à la troisième et non pas à la première personne), publié en 1953, que le narrateur mentionne, lors des retrouvailles chaleureuses à Paris de José avec Carmen, son vieil ami chauffeur et tombeur de femmes de la Route de Didier, qu'il n'avait pas vu depuis sept ans : « sept ans, en effet, depuis son départ de la Martinique, en 39, par "le premier convoi". C'est à Antibes qu'avait débarqué le bataillon... » (La Fête à Paris, 31).

Il y a donc eu au moins deux transpositions de cet épisode du "premier convoi» historique des mobilisés martiniquais vers la France en 1939, dans l'œuvre fictionnelle semi-autobiographique de Zobel. Le pionnier grand et costaud, ex-pêcheur de la nouvelle, ne ressemble guère au chauffeur du roman - «si racé, si gaillard » $(R C N, 256$; mais il a « engraissé » quand José le retrouve à Paris, FàP, 30). Mais si Zobel a fait passer l'accent du personnage au convoi, dès le titre de la version $C$ de la nouvelle, c'était, me semble-t-il, dans une volonté de retoucher sérieusement le portrait de la protagoniste développé dans les versions précédentes. Il s'agissait, en quelque sorte, de «sauver la réputation de la femme à soldat Ferdinise ", en effaçant l'épilogue avec le caractère répétitif de ses nuits d'amour à la recherche de l'homme idéal. Car, à la réflexion, le titre « Pionnier d'une nuit », n'était guère justifié dans les versions $A$ et $B$, en raison du caractère itératif de ces rencontres, qui aurait justifié de mettre au pluriel ces "pionniers d'une nuit ", même si le narrateur empathique portait ces répétitions au

Continents manuscrits, 13 | 2019 
compte de la sentimentalité idéaliste de Ferdinise plutôt qu'à la réalité sociale d'une jeune femme démunie, recourant à la prostitution pour survivre avec son bébé. Aucune autre activité rémunératrice n'est mentionnée dans le récit.

Quoiqu'il en fût, le scénario de la seconde partie de la nouvelle change radicalement dans la version C qui, en effaçant l'épilogue des versions précédentes, justifiait le singulier du titre d'origine en ne décrivant que la rencontre avec le pionnier Isidore, et en augmentant (sans doute par compensation) la partie consacrée à la recherche éperdue d'Isidore par Ferdinise, dans une ville affolée par le "premier convoi » de mobilisés en partance.

De nombreux détails qui soulignaient la sensualité des rapports de Ferdinise avec son/ ses amant(s) sont supprimés, au profit de nouvelles descriptions de la ville en ce jour mémorable. Zobel retouche plusieurs passages même dans la première partie du texte, qui n'avait subi que de légères altérations stylistiques dans la version $B$, et qui sont bien plus développées maintenant. Ainsi, la rencontre initiale des protagonistes à la sortie de la caserne est-elle évoquée dans une formulation poétique nouvelle, très auctoriale : « Il y avait dans l'air de ces espèces de paillettes sombres à quoi l'on s'aperçoit que le soir commence à descendre » $(C, 1)$, suivie de plusieurs images de Ferdinise, focalisées par un Isidore "appréciateur » de son physique "craquant ", déjà mentionnées en partie plus haut:

Le pionnier la regarda des pieds à la tête. / Il avait tout de suite aimé cette voix qui ressemblait à des trilles. Du visage, il ne retint que le regard pareil à celui des petites filles qui ont l'air timide et qu'on dit effrontées. Et le corps, sous l'indienne fleurie de la robe courte et décolletée, lui donna aussitôt un peu comme l'envie qu'inspire le pain doré : de le presser avec les deux mains pour le plaisir de voir et d'entendre craquer la croûte. $(C, 1)$

Parallèlement aux efforts d'embellissement d'éléments du cadre spatio-temporel de l'action, qui relèvent à la fois du lissage stylistique et d'une représentation de réalités sociales et culturelles d'ordre historique, l'auteur manie la gomme pour corriger le portrait de Ferdinise, développé dans la version précédente. Disparaissent : «le geste de fausse pudeur » quand elle accepte qu'Isidore la suive, et le commentaire, fort peu catholique, des versions A et B, sur « le lit » qui, en raison des images saintes placées juste au-dessus " avait tout l'air de quelque autel destiné à on ne sait quels sacrifices " (B et $C, 1)$; disparaît également l'initiative d'une Ferdinise qui d'emblée «tourmentait de baisers " la tête du soldat encore en train de se déshabiller, ainsi que la phrase finale de la version $A$, conservée en $B$, décrivant Ferdinise «blottie contre le corps athlétique du soldat » et lui parlant "d'une voix faible et câline" (A et B, 3), et les deux paragraphes décrivant «la nuit d'amour et d'ivresse pour Ferdinise » et «sa crise de sentiment au moment de l'au-revoir » (A et B, 4). Plus symptomatiques encore de cette nouvelle représentation de la protagoniste est bien sûr le gommage de l'allusion à la " paye » de l'acte sexuel de la nuit, par le soldat lambda que Ferdinise a arrêté dans la rue $(B, 6)$, ainsi que tout l'épilogue avec Ferdinise ramenant «dans sa chambre de misère » quelque «beau gars, grand et fort " dans les bras duquel elle retrouverait Isidore, l'homme qu'elle avait élevé à « son idéal " - mais « nouveau gars » dont elle devait attendre aussi le petit « cadeau » sous forme de billet au petit matin (A et B, 7-8).

En lieu et place de cette Ferdinise récidiviste de l'acte de chair, motivée par son sentimentalisme exaspéré - et néanmoins rétribué - Zobel ajoute des détails sur l'itinéraire de ce "premier convoi ", réuni Place de la Mission, et sur Ferdinise s'épuisant à acheter des petits cadeaux à l'instar des autres femmes à la recherche d'un 
mobilisé sur le départ et - comme dans les versions $\mathrm{A}$ et $\mathrm{B}$ - ne réussissant pas à retrouver son Isidore. Et le narrateur de la version C, d'introduire le passage nouveau suivant :

La sueur, qui coulait en gouttelettes à son front, à la lisière des cheveux, et coulait en minces filets le long de ses tempes et de son cou, avait mouillé l'encolure de sa robe, et elle sentait ses deux seins comprimés dans son soutien-gorge, comme des légumes - concombre ou aubergine - qui, dans une marmite couverte, rendent suffisamment d'eau pour assurer leur cuisson. $(C, 6)$

On peut estimer que la comparaison entre attributs féminins et légumes ci-dessus ne fait pas honneur au style généralement si maîtrisé de l'écrivain, et que les descriptions nouvelles, introduites par Zobel dans la page suivante pour évoquer les mouvements de la foule autour du convoi sont mieux inspirées. Puis, au lieu de laisser le lecteur sur la belle image finale des versions A et $B$, de Ferdinise "plaquant son corps souple et nerveux contre la poitrine chaude du soldat » de telle ou telle nuit (A et B, 7-8), le narrateur de la version $C$ conclut sur une Ferdinise pitoyable, perchée vers midi dans la foule sur un trottoir de Fort-de-France où «le soleil était si chaud et la musique des clairons si éclatante au-dessus de la cohue, qu'elle dut se tenir la tête de ses deux mains pour l'empêcher d'exploser » $(C, 8)$.

\section{Conclusions}

On conviendra que la version C de la nouvelle rebaptisée «Le premier convoi » se termine par une note d'un réalisme qui n'a rien du merveilleux caractérisant une grande partie de la prose de Zobel - alors qu'on peut l'entrevoir dans les versions précédentes. Comparée à celles-ci, la révision du texte semble consister surtout à punir la protagoniste pour sa nuit avec le pionnier, en transformant sa recherche d'Isidore le long du convoi de mobilisés en une espèce de chemin de croix ${ }^{20}$. Si le sentimentalisme de Ferdinise reste évident, ses actes spontanés de chaleur et de sensualité, et surtout le caractère répétitif de ses nuits avec des soldats est effacé, même s'il reste implicite dans le mode de sa rencontre avec Isidore au début du récit. L'impression domine que les précisions sur le contexte de la matinée du départ et les embellissements stylistiques remplacent les aspects sensuels développées dans les versions A et B. En fin de récit dans la version C, la fille - sinon «de joie", du moins de "plaisir»- Ferdinise du «Pionnier d'une nuit », se retrouve littéralement seule et en souffrance dans le Fortde-France chaotique du « Premier convoi ».

Plutôt qu'une maturation psychologique de l'écrivain, cette révision du portrait de la protagoniste semble relever d'un choix - sans doute conscient - de nature socioculturelle. En reformatant le récit pour publication dans Présence Africaine en 1978, Zobel a clairement déplacé le projecteur de la chambre initiale avec son lit, «autel destiné à on ne sait quels sacrifices » (mais on sait à quel " péché »...), vers le contexte d'une journée historique où Ferdinise rejoint les cohortes de braves femmes soucieuses d'apporter un petit réconfort - non plus sur l'oreiller - à leurs non moins braves hommes, en partance pour la guerre en France.

À l'époque de cette réécriture du recueil Laghia de la mort, Zobel avait quitté le Sénégal et considérait un retour en Martinique avant d'opter pour son installation définitive dans sa maison bien-aimée des Cévennes. Peut-être craignait-il que le portrait d'une Ferdinise, si câline avec ses compagnons d'une nuit, qu'il avait chaleureusement brossé 
en 1940 et conservé en 1946, serait offensant pour les lecteurs - et surtout les lectrices de Martinique ? La question n'est pas simple, car le portrait d'Émilienne dans « Il était un petit navire ", une autre protagoniste de mœurs légères, a été beaucoup moins retouché. Il est vrai qu'elle n'était pas encombrée par un bébé à nourrir et qu'on ne la voyait pas avec de la compagnie masculine dans sa chambre ou dans les cabines des bateaux que quelque officier, rencontré le soir au Select Tango, lui faisait visiter.

Il est probable que beaucoup des lectrices d'aujourd'hui préfèrent le genre de femme étonnamment libre pour l'époque que Zobel a décrit avec Émilienne. Et probable aussi que des lecteurs masculins, s'ils se laissent emporter par la fiction, se verraient peutêtre volontiers pour une nuit aussi, dans les bras de la Ferdinise des versions de 1940 et de 1946, plutôt que celle de 1978 .

\section{ANNEXES}

Annexe 1. Trois versions (A, B, C) d'une nouvelle de Joseph Zobel

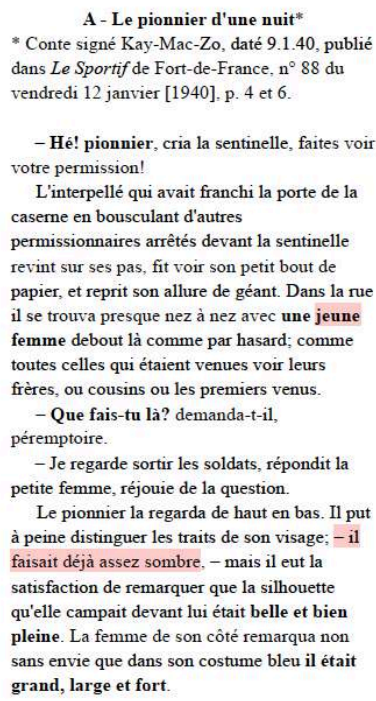

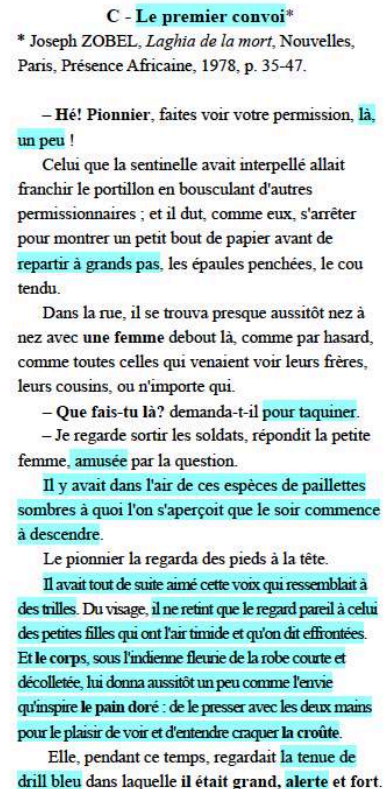


- Oủ habites-tu? reprit l'homme.

- Là-bas, tout près.
- Je viens avec toi?

Elle eut un petit geste de fausse pudeur et dit:

- Si tu veux... C'est pas loin..

- Si tu veux... C'est pas loin... Ils firent quelques pas sans rien se dire, le sold
enchanté de la manière décisive dont commençait sa nuit. Puis ils s'enfoncèrent, la femme devant, ans un couloir étroit, sombre et tortueux parmi un pâté de taudis en bois de caisse. Elle tâta une porte, ouvrit et entra la premiè

-Excusez-moi, dit-elle... je n'ai pas de pétrole.

La chambrette était éclairée par une lueur terne.

-Vous y voyez suffisamment? Ajouta-t-elle à voix basse. Asseyez-vous.

Le soldat s'affaissa sur le bord du lit en fer qui grinça, et dont le drap malgré l'insuffisance de

lumière, semblait très propre.

Petite et basse, la chambre était entièrement tendue de papier journal et de feuilles de

catalogues. Au quatre coins les poutres ayant perdı l'équilibre produisaient un effet de contorsion. Le lit qui occupait juste une paroi au milieu de laquelle allumait une veilleuse à l'huile entourée d'images saintes, avait tout l'air de quelque autel destiné à on ne sait quels sacrifices. A un petit espace, tout près, ne sait quels sacrifices. A un petit espace, tout pres, deux chaises,

Ce décor de débine n'avait pas frappé outr nesure l'homme qui demeura assis, les coudes plantés sur ses cuisses. Puis il enleva son casque épais et allait le poser sur le plancher, quand il vit près de ses pieds parmi des hardes, une tête d'enfant. Inconsciemment il eut un haut le corps de surprise.

- Vous avez un enfant? Demanda-t-il incontinent.

- Oui, répondit la jeune femme d'une voix froide; il a onze mois

Et, pressentant une nouvelle question:

Son père... ne s'occupe pas de lui.

L'autre se debarrassa nonchalamment de ses godasses qu'il laissa tomber lourdement, puis se leva et commença à se déshabille.

Comment t'appelles-tu chéri, demanda-t-ell en pressant entre ses mains la tête du soldat, qu'elle tourmentait de baisers.

- Isidore; mais chez moi on m'appelle Dodore. Tu sais, je faisais la pêche avant la mobilisation; je gagnais de l'aroent. Dans mon village on m'aimat beaucoup.

Mon nom est Ferdinise, dit-elle, mais ceux qui m'aiment m'appellent Ninise. Ton nom est plus qui m'aiment $\mathrm{n}$

Blottie contre le corps athlétique du soldat, Ferdinise continua d'une voix faible et câline, avec un accent plein de tressaillements, qui isait plus que ses paroles.

- Si tu veux, chaque soir tu viendras me voir. J t'aimerai comme je t'aime ce soir, de toute mon
- Où habites-tu? reprit l'homme.

-Ici, tout près.

- Je viens avec toi?

Elle ent un geste de fausse pud

-Si tu veux... C'est pas loin...
Ils firent quelques pas sans rien se dire, le soldat

Ils firent quelques pas sans rien se dire, le soldat
enchanté de la manière décisive dont commencait sa

enchanté de la manière décisive dont commençait sa

nuit. Puis ils s'enfoncerent, la femme devant, dans un couloir étroit, sombre et tortueux parmi un pâté de taudis en bois de caisse.

Elle tâta une porte, ouvrit et entra la première.

Excuse-moi, dit-elle... je n'ai pas de pétrole.

La chambrette était éclairée par une lueur terne.

-Tu vois suffisamment? ajouta-t-elle à voix basse. Assieds-toi.

Le soldat s'affaissa sur le bord du lit en fer qui grinça. Le drap, malgre l'insuffisance de la lumière, semblait très propre.

Petite et basse, la chambre était entièremen endue de papier journal et de feuilles de catalogues Au quatre coins, les poutres ayant perdu l'équilibre, les cloisons se contorsionnaient. Le lit qui occupait juste une paroi au milieu de laquelle allumait une veilleuse l'huile entourée d'images saintes, avait tout l'air de quelque autel destiné à on ne sait quels sacrifices. Tou près, deux chaises, dans la pénombre, intercalaient un petite table.

Ce décor n'avait pas frappé outre mesure lhomme qui demeura assis, les coudes plantés sur ses cuisses. Puis il enleva son casque épais et il allait le poser sur plancher, quand il vit près de ses pieds parmi les hardes, une boule noire, une tête d'enfant.

Inconsciemment il eut un haut le corps de surprise.

- Vous avez un enfant?

- Oui, répondit la jeune femme d'une voix froide; a onze mois.

Et, pressentant une nouvelle question

L'autre se débarrassa bon [sic]

de ses godillots qu'il laissa tomber avec fracas, pui se leva et commença à se déshabille.

- Comment t'appelles-tu, chéri, demanda-t-elle en pressant entre ses mains la tête du soldat, qu'elle tourmentait de baisers.

- Isidore ; mais chez moi on m'appelle Dodore. Tu sais, je faisais la pêche avant la mobilisation-je gagnais de l'argent. Dans mon village on m'aimait beaucoup.

- Mon nom est Ferdinise, dit-elle, mais ceux qui m'aiment m'appellent Ninise. Ton nom est plus doux, Dodore.

Blottie contre le corps athlétique du soldat, Ferdinise continua d'une voix faible et câline, avec un accent plein de tressaillements, qui disait plus que ses paroles :

que ses paroles :
- Si tu veux, chaque soir tu viendras me voir, je -Si tu veux, chaque soir tu viendras me voir, je
t'aimerai comme je taime ce soir, de toute mon âme..
- Ou habites-tu ? reprit-i1.

- Ici, tout près.

- Alors je viens avec toi, tu veux bien ?

Elle voulut paraitre indifférente, mais sous le regard qu'il maintenait sur elle, elle dit avec le même air de plaisanter :

- Si tu veux; c'est pas loin.

Ils firent quelques pas sans rien dire, le soldat enchanté de la manière décisive dont la nuit s'ouvrait à

La rue se dirigeait vers le centre de la ville en charriant des vieilles autos et des camionnettes poussives, des piétons mal habillés, des bicyclettes zigzagant et jouant sans cesse de leur avertisseur. Puis, la femme le précédant, ils enfilèrent, parmi des baraque dont quelques unes avaient déjà allumé leur lampe à pétrole. un couloir étroit qui se tordait au fur et à mesure qu'on avançait. Le genre de passage où, d'instinct, l'on feutre ses pas. Elle s'arrêta bientôt, tàta une porte avec une clé qui sans doute été gardée dans sa main, ouvrit entra la première.

- Excusez-moi, dit-elle ; j'ai oublié d'acheter du

Et encore plus bas, comme pour ajuster sa voix à l'obscurité de la pièce : « Mais je crois qu'on y voit tou de même. Assieds-toi.

Le lit occupait juste une paroi au milieu de laquelle, sur une petite étagère, était allumée si faiblement qu'il ne l'avait pas remarquée en entrant, une veilleuse à l'huile entourée d'images de piête.

Tout près, deux chaises, dans la pénombre, encadraient une petite table.

Ce décor n'avait pas beaucoup surpris l'homme qui demeura assis, le torse en avant, les coudes plantés sur ses cuisses.

Puis il enleva son casque épais, et il allait le poser sur le plancher, quand il vit près de ses pieds, sur des chiffons jetés à même le sol, la tête un peu plus sombr d'un bẻbé.

sursaut.

est ton enfant ? demanda-t-il avec un léger

- Oui, dit-elle d'une voix froide; il a onze mois Et, devançant une nouvelle question :

Son père... ne s'occupe pas de lui.

Il se débarrassa de ses godillots qu'il laissa tomber lourdement, puis, se levant, commença de se déshabiller

Comment t'appelles-tu ? demanda-t-elle en prenant la tête entre ses mains.

- Isidore; mais chez moi on dit Zido... Tu sais, je

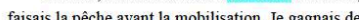
l'argent Dans mon village on - Moi, c'est Ferdinise, Mais t'as qu'à dire Nise tout le monde me connait ici

Ils baissèrent encore la voix, car on entendait les gens marcher et parler dehors, dans la ruelle - Si tu veux, reprit-elle, tu peux venir chaque fois que tu sors, chaque soir, même 
âme, toujours, rien que toi. Moi, je déteste le hommes de la ville. Ils n'ont pas de cœur, et quand ils couchent avec une femme qui a un enfant, ils n'ont même pas la pitié de lui donner de quoi acheter du toloman pour le pauvre petit. Toi tu es soldat, je n'ai pas besoin que tu me donnes rien. Pas même mes loyers. Au contraire, tu es soldat. Et puis, après la guerre, quand tu vas redevenir pecheur, on va se voir souvent, chaque fois que tu pecherr, on se vo soisson. Je ferai toutes que viendras cons pour se voir. Et daillens la jonte resteras coucher le soir. Tu seras chez toi. Hein,
veux ?

L'homme qui commençait à s'endormir

l'attira plus près, contre lui, et répondit du ventre. !- En-hen!

Et elle, pour s'endormir aussi, posa sa tête ivre de rêve entre l'épaule tiède et le menton chatouillement piquant de l'amant, et lui sangla le corps d'un bras caressant.

La nuit avait vite passé ; nuit d'ivresse et d'amour pour Ferdinise, simple nuit de plaisir pour Isidore; et au petit jour ce dernier avait rallié la caserne. Ferdinise alors fut prise d'une crise de sentiment au moment de l'au-revoir, le suppliant de revenir le soir, le conjurant d'avoir pitié de son. idéal auquel elle avait renoncé de désespoir, mais dont elle sentait la réalisation possible mainten Lui avait promis.

Plus tard, elle avait trouvé sur sa table un billet

de cent sous et cela l'avait beaucoup émue.

\section{Le bon soldat:}

Ayant confié son enfant à une voisine, Ferdinise munie de son billet partit pour le marche. Mais la jeune femme n'était pas plutôt sortie que le spectacle insolite de la rue effraya son coeur. L'animation ce matin-là était faite du va-et-vient d'une foule de soldats en uniforme de drap. OhElle avait bien entendu parler de ces uniformes qu'on préparait pour ceux qui devaient partir, mais elle n'y avait plus pensé

Alors elle s'arrêta net, et regarda longtemps passer en tous sens les soldats, avec des escortes de parents et d'amis; ceux rapprochés de la ville et qui, à la nouvelle brusque du départ avaient eu le temps d'accourir pour porter des cadeaux et embrasser les

Ferdinise debout regardait la tête vide. De hommes, des hommes en uniforme de drap réséda! Ils étaient tiraillés par-ci, par-là, vers les bistrots, tirés encore par les femmes qui ne voulaien pas se détacher d'eux et qui les embrassaient avec transport et désolation. Ferdinise alors repartit vers la caserne, croisant des soldats, certain

enthousiastes.

Alors elle eut l'idée que, comme les autres il était sorti boire avec des copains, et elle partit vers le boulevard. Avec les cinq francs elle acheta pour lu un demi-litre de rhum, une grappe de belles oranges, et enfin quelques médailles de divers saints, puisque presque toutes les femmes en achetaient pour offrir à ceux qui partaient. de la ville. Ils n'ont pas de ceurr. et quand ils couchent avec une femme qui a un enfant. ils n'ont même pas la pitié de lui donner de quoi acheter du toloman pour le pauvre petit. Toi, tu es soldat, je n'ai pas besoin que t me donnes rien. Pas même mes loyers. Au contraire, tu es soldat. Et puis, après la guerre, quand tu vas redevenir pecheur, on va se voir souvent; chaque fors rai toutes tes Tt deilleurs si n joumce ost top c

'endormir l'attira plus près, contre lui, et répondit du ventre:

-Han, han

Et elle, pour s'endormir aussi, posa sa tête ivre de êve entre l'épaule tiède et le mento corps d'un bras caressan

La nuit avait vite passé. Nuit d'amour et d'ivresse pour Ferdinise, simple nuit de plaisir pour Isidore; $e$ au petit jour ce dernier avait rallié la caserne. Ferdinis alors fut prise d'une crise de sentiment au moment de l'au-revoir, le suppliant de revenir le soir, le conjurant d'avoir pitié de son idéal auquel elle avait renoncé de désespoir, mais dont elle sentait la réalisation possible maintenant. Lui avait promis.

Plus tard, elle avait trouvé sur sa table un billet de cent sous, ce qui l'avait beaucoup ému.
... toujours, rien que toi. Moi, je déteste les hommes chatouilleusement piquant de l'amant, et lui sangla le

...Moi, tu sais, ce n'est pas faire la vie, que j'aime. D'ailleurs je déteste les hommes qu'on ne voit qu'une fois et qui vous laissent tomber aussitôt, là même, parce qu'on a un enfant... Ça ne pense qu'à s'amuser ! Mais je ne dis pas ca pour toi. Toi, tu es un soldat ; ce n'est pas la même chose... Mais après la guerre, quand tu vas reprendre ton métier to pirogue, tes filets, on va se voir souvent chaque fois que tu viendras apporter du poisson au marché Je ferai toutes tes conssions pour quis a d a d' Tu seras chez toi. ici. Hein, tu veux?

Il commençait à s'endormir et depuis un instant ne l'écoutait plus. Pour toute réponse, il l'attira plus près, contre lui

La nuit fut pourtant vite passée et le billet de cent sous que Ferdinise trouva sur sa table, après qu'il fut parti, confirma la gentillesse et la délicatesse de celui avec qui elle se voyait déjà recommencer une vie

nouvelle

Aussi, comme pour marquer ce nouveau début, et comme on fait un sortilège, elle décida qu'elle irait au marché et qu'elle achèterait, pour casser le billet, des choses qui portent bonheur : des oranges, dont les graines, si on les garde, attirent l'argent ; du riz, qui est l'abondance même ; du sel, dont une case ne devrait jamais manquer.

Le ménage fut vite fait : l'important est que le lit soit bien couvert.

Quand on quitte chez soi, on ne sait jamais : on peut
bien couvert. Quand on quitte chez soi, on ne sait jamais : on peut
se trouver pris d'un malaise à n'importe quel moment, se trouver pris
n'importe où,

...et ce serait une honte d'être ramené chez soi par des

Le bon soldat!

Alors Ferdinise confia son enfant à une voisine et, son billet au creux de sa main, partit pour le

Mais la jeune femme n'était pas plutôt sortie que le spectacle insolite de la rue effraya son coeur. L'animation de ce matin-là était faite d'un va-etvient de soldats en uniforme de drap. Oh! Elle avait bien entendu parler de ces uniformes qu'on préparait pour ceux qui devaient partir. Elle s'arrêta net et regarda longtemps passer en tous sens des soldats escortés de parents et d'ami ceux rapprochés de la ville et qui, à la nouvelle brusque du départ, avaient eu le temps d'accourir pour apporter des présents et embrasser les leurs. Ferdinise, debout, regardait la tête vide. Des hommes, des hommes en uniforme de drap réséda!

Ils étaient tiraillés par-ci, par-là, vers les bistrots. tirés encore par les femmes qui ne voulaient pas se détacher d'eux, et qui les embrassaient avec transport et désolation

Ferdinise alors repartit vers la caserne, croisant des soldats, fouillant des yeux tous les visages dhommes en uniforme

Puis elle eut l'idée que, comme les autres, celui qu'elle cherchait était sorti boire avec des copains, et elle partit vers le boulevar

Avec les cinq francs, elle acheta pour lui un demi-litre de rhum, une grappe de belles oranges, et enfin quelques médailles en aluminium de diver saints, puisque presque toutes les femmes en achetaient pour offrir à ceux qui partaient. gens qui vous auront ramassée et qui s'apercevraient que vous n'avez même pas fait votre lit avant de sortir. Le plus long etait de peigner ses cheveux et de les enrouler en " petits choux » maintenus par des épingles.

Elle fut vite prête. Il ne lui resta qu'à prier une un saut au marché », précisa-t-elle; et la voilà partie. Mais à peine eut-elle quitte la rue Brithmer avec ses vèicules titubant sur les fondrières, ses marchands dont on eût dit les rites du quartier pour le commencement du jour, qu'elle remarqua que la ville avait pris un aspect insolite et qui la troubla. L'animation habituelle avait été renforcée d'un va-et-vient de soldats en uniforme de drap couleur caca d'oie. Oh! elle avait bien entendu parler de ces uniformes qu'on préparait pour ceux qui devaient partir, ceux du premier convoi. Elle s'arrêta net et regarda longtemps en tous sens des soldats escortés de parents et d'amis

Elle regardait tellement qu'elle semblait ne plus

pouvorr bouger

Des hommes, des hommes en uniforme.

IIl lui semblait qu'au fur et à mesure que sa tête se vidait.

Des hommes en uniforme tiraillés par-ci, par-là, vers des bistrots, tirés encore par les femmes qui ne voulaien pas se détacher d'eux et qui les embrassaient avec transport et désolation

S'animant alors tout à coup, elle repartit en direction de la caserne, croisant des soldats, interrogeant des yeux tous les groupes d'hommes en uniforme réséda voisine de garder l'enfant, « juste le temps que je fasse ambulants, ses gosses dépenaillés, et toutes les scènes 
Et son petit cadeau à la main elle errait, fouillant des yeux tous les bistrots. N'allait-elle pas le trouver? Langoisse l'empoigna encore plus for, séparation, et non à celle plus cruelle d'une sorte disparition sans adien Toujours des hommes en uniforme, et qui
allaient partir, des homme, plus beaux, par leur enthousiasme ou leur mélancolie. Tellement d'hommes, grands et beaux, qu'elle finit peu à peu douter de pouvoir reconnaître celui qu'elle chercha Vraiment à la lueur de la veilleuse avait-elle bien regardé ses traits, ou ne l'avait-elle pas vu autreme à travers son trouble et sa griserie? Le reconnaîtritelle? Oh non, ce n'était pas bien sûr. Pourtant elle continua à aller et venir cherchant avec une attention désespéré

Mais si elle demandait pour lui?

Elle aborda un du départ qui lui semblait sympathique

Est-ce que vous ne connaissez pas un solda qu'on appelle Isidore?

- Isidore comment? reprit le solda

- Isidore ... il est grand, costaud ... bégaya-t elle, affolée, en se crispant les doigt.

-Eh oui! Tu crois ça comme ça? Un soldat, ça a comme tout le monde; et ça appartient à une compagnie par-dessus le marché ! Eh bien, ma doudou, dans les conditions qu'on par s'il ne t'a pas payée hier soir, ton Dodore!

Ferdinise voulut se ressaisir et invectiver l'impitoyable, mais elle était si brutalement atteinte de désespoir que la force lui manqu

et elle se mit à sangloter en marchant avec égarement. Mais... Brusquement un son de clairon alerta tout le monde vers la grande place où devait passer le convoi. Elle prit aussi la course. La place était déjà comble avec une foule
débordant jusquiaux faites des autobus. Seulement la musique qui s'eloignait, et le bruit sourd des gros souliers lui parvenaient, et quand elle put trouver un endroit d'où elle pouvait voir, la moitié de la longue colonne massive avait défile

Elle ne vit pas Isidore.

Plus d'un soir Ferdinise s'en alla attendr devant la caserne la sortie des soldats, pour conduire dans sa chambrette de misere un beau gars grand et fort. A la lueur de la petite veilleuse Thuile, quelle allumait routinierement en implorant la pitie des saintes images, elle pleurair dans le 14 en fer grinçant, au pied duquel dormait son petit, pour que le soldat qui etait couche contre elle 'aimat au-dela de la guerre.

C'était son ideal. Cetait la recherche naĩve et ardente de cet ideal qui, chaque soir renouvelait dans son lit un homme indiferent quelle amait avec fièrre et le désespoir de tous ses sens et qu'elle ne pouvait jamais voir plus d une nuit. demandit an mâte assoupi: - Tu n'as pas connu un

ionnier nomm

Isidore? On l' appelait plutôt Dod

- Il était grand, large, fort et beau. Il avait...

Alors un tressaillement la raidissait dans toute sa chair, et elle plaquait son corps souple et nerveux contre la poitrine chaude du soldat. [Fin]

(Archives de Martinique. Transcription par Charles Scheel, juillet 2019)
Et, son petit cadeau à la main, elle errait, sher? L'anter elle avait pensé d'abord à la douleur de la séparation et non à celle plus cruelle d'une sorte de disparition et non a celle
sans adieu.

Toujours des hommes en uniforme, et qui allaient partir! Des hommes, plus beaux par leur enthousiasme ou leur mélancolie.

Tellement d'hommes, grands et beaux, qu'elle finit par douter de pouvoir reconnaitre celui qu'elle cherchait. Vraiment, à la lueur de la veilleuse, avaitelle bien regardé ses traits, ou ne l'avait-elle pas vu autrement à travers son trouble et sa griserie? Le reconnaitrait-elle? Oh non, ce n'était pas bien sûr Pourtant elle continua d'aller et venir, cherchant avec une attention désespéré

Mais si elle demandait?

Elle aborda un du départ qui lui semblait sympathique.

Est-ce que vous ne connaissez pas un soldat qu'on appelle Isidore?

- Isidore comment? répliqua le soldat.

- Isidore .... il est grand, costaud . . . bégaya-telle, affolée, en crispant ses doigts

- Eh ouil Tu crois ça comme ça? Un soldat, ça a un nom et un prénom comme tout le monde; et ça appartient à une compagnie par-dessus le marché Eh bien, ma doudou, dans les conditions qu'on part, s'il ne t'a pas payée hier soir, ton Zidore! . . Ferdinise voulut se ressaisir et invectiver l'mpitoyable, mais elle était si brutalement atteinte dans son espérance que la force lui manqua.

Elle fondit en larmes et partit, tout éperdue. la petite ville vers la place où devait passer le convoi. Elle prit aussi la course.

La place était déjà comble, avec une foule débordant jusqu'aux faîtes des autobus.

Seuls la musique qui s'éloignait, et le bruit sourd des gros souliers lui parvenaient, et quand elle pur trouver un endroit d'où elle pouvait voir, la moitie de la colonne massive avait défile.

Elle ne vit pas Isidore.

Plus d'un soir Ferdinise s'en alla attendre devant la caserne la sortie des soldats pour conduire dans sa chambre de misère un beau gars grand et fort. A la lueur de la petite veilleuse à Thuile, qu elle allumait routinierement en imploran la protection des saintes images, elle pleurait dan le lit en fer grinçant, au pied daquel dormait son petit, pour que le soldat qui était couché contre Cle lamara au-dela de la guerre.

C'etait son ideal. C'était la recherche naive et ardente de cet ideal qui, chaque soir mettait près d'elle un homme indiferent qu'elle aimait avec fièvre et le désespoir de toute sa tendresse et qu'el ne pouvait jamais voir plus d'une nuit. Parfois pourtant, grisee et rèveuse, elle
demandait au mâle assoupi-

- Tu n'as pas connu un pionnier nomm

Isidore? On l'appelait plutôt Dodore

- Non, grognait l'autre, évasif

- Il etait grand, large et fort. II était beau!
Puis, lui vint l'idée que, comme les autres, celui qu'elle cherchait était sorti boire avec des copains ; et elle toura vers le boulevard, mais aussitôt elle pensa que, du elle ne le verrait longuement si elle parvenait à le rencontrer, et qu'il valait mieux par consépuent, se munir rencontrer, et qu'il valait mieux, par consequent, se
à lavance de ce qu'elle pourrait lui offir en cadeau. Avec les cinq francs, elle acheta donc un demi-lite de rhum, une grappe de belles oranges, et aussi quelques médailles en aluminium à l'effigie de divers saints dont elle savait la renommée pour ce qui est de conjuration des dangers et la protection en cas de péril. Toutes les femmes en achetaient par douzaines pour distribuer à ceux qui partaient

Son petit cadeau à la main, elle errait, s'arrêtan devant chaque café, faisant de temps en temps une incursion dans ces petits bars sombres où l'on semble se cacher pour boire. Et brusquement, par instants, elle se demandait si elle pourrait même le reconnaître ; et ce doute, certes on ne peut plus absurde, déclenchait dans sa tête et ses entrailles, une panique que venait aussitôt refrèner le visage ou la carrure qu'elle apercevait là-bas au coin de la rue ou en entrant dans un magasin

La sueur, qui coulait en gouttelettes à son front, à la lisière des cheveux, et coulait en minces filets le long de ses tempes et de son cou, avait mouillé l'encolure de sa robe, et elle sentait ses deux seins comprimés dans son soutien-gorge, comme des légumes - concombre ou aubergine - qui, dans une marmite couverte, rendent suffisamment d'eau pour assurer leur cuisson Partout, des hommes en uniforme, et qui allaient partir

Quelque chose de pathétique dans leur résignation, dans leur mélancolie ou même dans leur enthousiasme, et qui les rendait magnifiques

Et, plus d'un qui ressemblait, à s'y méprendre chaque fois, à Isidore.

Et pourquoi ne demanderait-elle pas tout simplemen Ne connaîtriez-vous pas un soldat qui s'appelle... ? Isidore comment?

- Isidore... II est grand... Oui, costaud ... bégaya-telle, affolée, se crispant des doigts $\mathbf{O n}$ l'appelle plutôt Zido.. Zido ? Et quel RIMA Elle dut abandonner : son cour se gonflait jusqu'à sa gorge et elle se mit à marcher, sans but, sans rien regarder, les yeux brouilles par les larmes qu'elle ne

pouvaat mème pas faire l'effort de cacher.

Brusquement, un clairon retentit, et ce fut aussito conme si mand lequel toute la ville accourait au lieu de fuir. Du côté de la Place de la Mission

Ferdinise avait oui dire, en effet, que le convoi passerait par la Place de la Mission.

Elle n'eut qu'à se laisser entrainer par le courant qui descendait vers la Place de la Missio La foule y était déjà mêlée comme les eaux de plusieurs affluents d'un fleuve en crue.

Il y avait, sur le toit des autobus en stationnement, des gens qui semblaient etre portes sur hing
Alors un tressaillement la raidissait toute, et comme pour rattraper un songe fugitif, elle plaquat son corps souple et nerveux contre la poitrine chaude du soldat.

[Fin]
Ballottée, jouant des coudes, haletante. Ferdinise

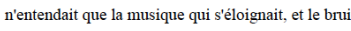
cadencé de gros souliers ; et quand elle eut réussi à gagner une place, plus de la moittié de la longue colonne massive etait passée.

D'ailleurs, de là où elle était perchée, c'est de dos qu'elle voyait marcher les uniformes.

Le soleil était si chaud et la musique des clairons si éclatante au-dessus de la cohue, qu'elle dut se teni la tête de ses deux mains pour l'empêcher d'exploser.

[Fin]

Annexe 2. « Partir », reportage de Kay-Mac-Zo, Le Sportif, n 80, Fort-de-France, 16.11.1939, p. 6 


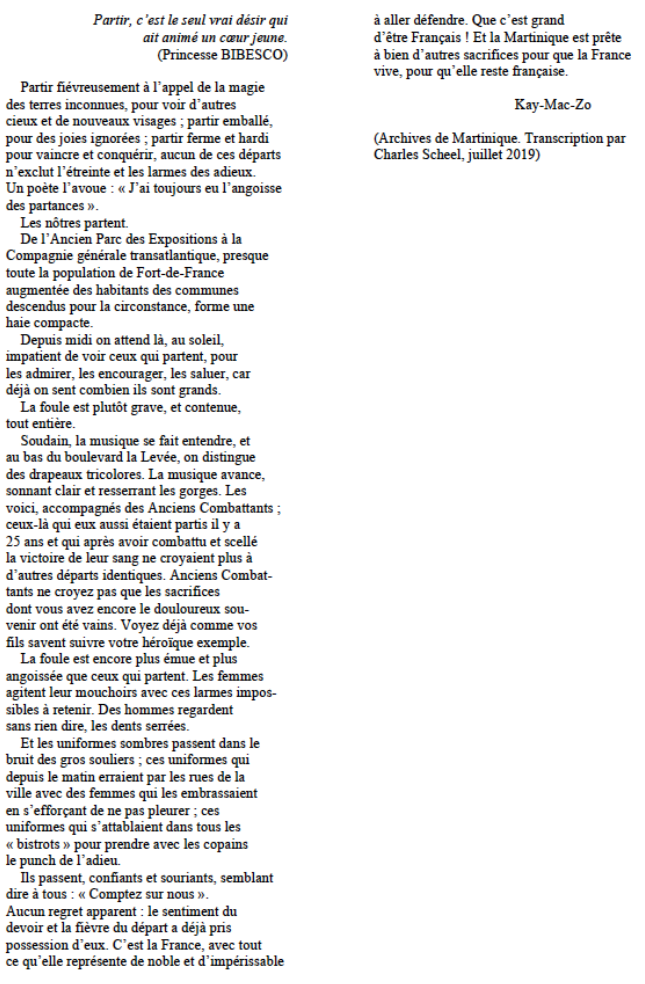

\section{NOTES}

1. Louise Hardwick, Joseph Zobel, Négritude and the Novel, Contemporary French and Francophone Cultures 51, Liverpool University Press, 2018, 275 p.

2. Charles Scheel, La Forge de Zobel, Paris, Scitep éditions, 2018, 221 p.

3. Joseph Zobel, Diab'-là (1945), Paris, Nouvelles Éditions Latines, 1946, 174 p.

4. Joseph Zobel, La Rue Cases-Nègres (1950), Paris, Présence Africaine, 1974, 311 p.

5. Seule «Bo-Bo-Bo-o », une nouvelle sur les débuts de la dissidence en Martinique, dédiée au gouverneur Georges-Louis Ponton, n'est pas reprise.

6. Il faut souligner que le titre est trompeur, car en fait il n'y a pas de combat à mort, contrairement au premier récit par Zobel sur ce thème de la danse de combat martiniquaise, «Geo Bamboula » (redécouvert récemment et paru dans La Forge de Zobel, 21-26).

7. Contrairement à M'man Tine, Cocotte a hérité de son grand-père béké, possède des terres et aurait même « de l'argent caché sous les fondations de sa case » (cf. Hardwick 2018, 108). Elle a donc les moyens de jouer un rôle social important dans la communauté, notamment en prêtant de l'argent à des jeunes entreprenants dans son entourage. Cet aspect généreux du personnage est contrasté par un caractère explosif et querelleur, illustré surtout dans une bataille hargneuse avec son cousin détesté, Sylva, pour le contrôle de l'eau - ressource précieuse dans ce coin le plus sec de Martinique.

8. L'appellation «conte» pour les récits courts domine dans la presse mais cède le pas à «nouvelle» en librairie.

9. Ce dernier conte n'avait pas été publié initialement dans Le Sportif, comme les deux autres, mais il a été rédigé à la même époque. Il est d'autant plus intéressant que sa première édition dans Laghia de la mort de 1946 offre une verdeur de vocabulaire dans les scènes d'affrontement entre mâles, qui sera grandement tempérée dans celle de 1978. 
10. Car le tout premier protagoniste féminin de Zobel publié était Justina, dans le conte éponyme paru dans Le Sportif du 12 octobre 1939, mais non repris en recueil. Justina est une belle jeune fille qui, après avoir connu une déception relationnelle "en ville", revient dans son village en affichant des airs hautains, encouragés par sa mère. Mais elle finit par se laisser séduire par Malijo, son ancien copain d'école : "elle entra dans le cercle de la bamboula et dansa pour lui avec une frénésie amoureuse et passionnée » (Forge, 34). Il s'agit donc d'un conte sur l'opposition villecampagne qui n'est pas contextualisé dans le conflit de 1939-1945, contrairement à l'histoire de Ferdinise qui paraît à peine deux mois plus tard (le 12 janvier 1940) et celle d'Émilienne quatre ans plus tard (le 2 mars 1944).

11. Notons au passage que le contact s'établit avec une facilité et un naturel admirables, sans la moindre allusion à une transaction " commerciale ", et sans aucun détail grivois sur les rapports sexuels qui restent implicites, comme entre Diab'là et Fidéline, les protagonistes du premier roman de Zobel en cours de rédaction à cette époque.

12. On aimerait savoir combien de femmes lisaient Le Sportif de Fort-de-France à cette époque.

13. Notons que ces termes sont inversés dans la version B : dans A, Ferdinise cédait à l'ivresse avant l'amour.

14. On peut apprécier la responsabilité du « chaque soir » qui « mettait un homme » dans le lit de Ferdinise. D'autre part, sauf erreur, cet "aimât" est la seule occurrence d'un imparfait du subjonctif dans le discours du narrateur de cette œuvre. Il paraît d'autant plus artificiel dans le contexte sociologique de l'histoire, que dans la réalité martiniquaise de l'époque, de tels protagonistes se seraient exprimés en créole.

15. Contrairement à Émilienne dans "Il était un petit navire », qui est tentée par une petite "crise sentimentale» au moment où elle se souvient de sa brévissime rencontre avec le beau Jimmy de Trinidad, avant de se reprendre et de retourner au Tango Sélect où elle rencontre ses aventures d'un soir (ou d'une nuit) et « fait sa vie » en ces temps difficiles de guerre - c'est-à-dire antan Robè pour la Martinique.

16. Nonobstant ces formules poétisées, le mode narratif de la nouvelle est bien plus sobre que le réalisme merveilleux des deux premiers romans que Zobel avait rédigés entre 1940 et 1946, Diab'là et Les Jours immobiles.

17. Il n'est peut-être pas anodin non plus, que cette nouvelle particulière ait été dédiée à Michel Reynoird Zobel, demi-frère de l'écrivain (toutes les dédicaces seront effacées dans le recueil de 1978).

18. La plus gênante concerne une assertion erronée surprenante dans le court paragraphe présentant l'auteur en quatrième de couverture (erreur reprise dans toutes les réimpressions depuis) : "Joseph Zobel est Martiniquais. Il a écrit plusieurs romans et nouvelles dès les années 50". Or il avait déjà publié deux romans et son premier recueil de nouvelles dans les années 40. La parution de Laghia de la mort en 1946 est d'ailleurs mentionnée dans le paragraphe précédent, de la même quatrième de couverture, mais dans une longue phrase tortueuse qui pourrait être amendée. Celle-ci met en avant La Rue Cases-Nègres, dont la réédition par Présence Africaine en 1974 a certainement promu « le rayonnement culturel de la Martinique » (et cela même avant le succès du film). Mais fallait-il pour autant ignorer Diabl'-là ?

19. Elle montrait en couverture un bois gravé d'André Couret, représentant deux lutteurs et un tambourier - illustration remplacée par une photo d'une plage et d'un morne de Martinique dans un nouveau format, bien plus petit.

20. Presque littéralement, car cette place que Zobel appelle « de la Mission », correspond à celle communément appelée "Place de la Croix-Mission", à côté du grand cimetière de Fort-deFrance, où se trouvait alors la gare d'autobus et de taxis-pays. Notons que d'autres noms de lieux sont mentionnés dans le reportage « Partir » (Annexe 2). 


\section{RÉSUMÉS}

La plus grande partie de l'œuvre littéraire de Joseph Zobel a été produite pendant les années 1939-1946 en Martinique et pendant les années 1947-1957 en France. Mais plusieurs romans et nouvelles ont été réédités dans des versions modifiées après son retour du Sénégal à la fin des années 1970. On s'intéresse ici à la nature des modifications apportées à une nouvelle particulière, publiée d'abord dans un journal martiniquais en 1940, puis dans le recueil Laghia de la mort, paru à Fort-de-France en 1946, et finalement dans la réédition du même recueil par Présence Africaine à Paris en 1978. Les changements apportés dans le portrait de la protagoniste sont particulièrement frappants dans cette dernière version.

Most of Joseph Zobel's literary work was produced during the years 1939-1946 in Martinique and between the years 1947-1957 in mainland France. But several novels and short stories were reissued in modified versions after Zobel's return from Senegal at the end of the 1970s. What is studied here is the nature of the modifications worked upon a particular short story which was published first in a Martinique newspaper in 1940, then in the collection Laghia de la mort printed in Fort-de-France in 1946, and finally in a revised edition of the same collection with Présence Africaine in Paris in 1978. The changes brought to the representation of the female protagonist are particularly striking in the latter version.

\section{INDEX}

Mots-clés : Zobel, Martinique, nouvelle, représentation de la femme, style, maturation, censure

Keywords : Zobel, Martinique, short story, representation of women, style, maturing, censorship

\section{AUTEUR}

\section{CHARLES W. SCHEEL}

Université des Antilles, Martinique 